\title{
Synthesis of Thioglycosides by Tetrathiomolybdate-Mediated Michael Additions of Masked Thiolates
}

\author{
Perali Ramu Sridhar, ${ }^{[a]}$ Kandikere Ramaiah Prabhu, ${ }^{[a]}$ and Srinivasan Chandrasekaran ${ }^{*[a, b]}$
}

Keywords: Carbohydrates / Domino reactions / Michael addition / Tetrathiomolybdate / Thiosaccharides

\begin{abstract}
An efficient one-pot methodology for the synthesis of thioglycosides in excellent yields under neutral conditions through the use of benzyltriethylammonium tetrathiomolybdate $\left[\left(\mathrm{BnNEt}_{3}\right)_{2} \mathrm{MoS}_{4} ; \mathbf{1}\right]$ as a sulfur-transfer reagent has been developed. The reagent 1 reacts with sugar halides to give sugar disulfides, which then undergo reductive cleavage in situ to provide the corresponding thiolates, followed by Michael addition to give the corresponding thioglycosides.
\end{abstract}

Further, the utility of this one-pot reaction in aqueous medium has been exemplified through the use of ammonium tetrathiomolybdate $\left[\left(\mathrm{NH}_{4}\right)_{2} \mathrm{MoS}_{4} ; 2\right]$. The application of this methodology has been extended to the synthesis of a variety of thiosugar analogues with excellent diastereoselectivity through inter- and intramolecular reactions.

\section{Introduction}

The chemistry and biology of thioglycosides have seen dynamic progress thanks to the favorable chemical and biological stability associated with them. ${ }^{[1]}$ S-Glycosides have additional advantages over C-glycosides, since an interglycosidic sulfur atom may act as a hydrogen-bond acceptor and could play an important role in ligand binding, especially in the cases of enzyme inhibitors ${ }^{[2]}$ and enzymeresistant scaffolds, ${ }^{[3]}$ and therefore new developments directed towards the synthetic and medicinal chemistry of thiosugars are important for carbohydrate drug design. ${ }^{[4]}$ Apart from this, thioglycosides are also useful synthons for the synthesis of C-glycosides through the RambergBäcklund rearrangement ${ }^{[5-7]}$ of O-glycosides by activation with NIS and AgOTf, ${ }^{[8]}$ and of 1-deoxyglycosides by treatment with Raney nickel. The available methods for the synthesis of thioglycosides utilize sulfur's more nucleophilic and less basic properties (relative to oxygen) to provide thioglycosides in an $\mathrm{S}_{\mathrm{N}}$ 2-type substitution process. ${ }^{[9]}$ The most commonly used methods include the use of isothiourea derivatives of sugars (in turn derived from the glycosyl halides and thiourea) as precursors, ${ }^{[10]}$ treatment of glycosyl halides with thiolate anions, ${ }^{[1]}$ or the reaction between a 1thioglycopyranose and an alkyl halide. ${ }^{[12]}$ Other methods are based on Lewis acid catalyzed glycosylation of thiols. By this approach, thioglycosides have been prepared by starting from 1,2-trans-glycosyl esters, ${ }^{[13]}$ trichloroacetimid-

\footnotetext{
[a] Department of Organic Chemistry, Indian Institute of Science, Bangalore 560012, India

Fax: (internat.) + 91-80-2360-0529

E-mail: scn@orgchem.iisc.ernet.in

[b] Jawaharlal Nehru Centre for Advanced Scientific Research, Bangalore, India
}

ates, ${ }^{[14]} 1$ - $O$-trimethylsilyl glycosides, ${ }^{[15]}$ and from glycopyranoses. $^{[16]}$ Thioglycosides are also prepared from carbohydrate sulfenates ${ }^{[17]}$ and by free radical addition of 1thiosugars to alkenes. ${ }^{[18]}$ Although there are a number of methods available for the synthesis of thioglycosides, a simple and user-friendly method is still needed.

\section{Results and Discussion}

In continuation of our efforts towards the synthesis of sulfur-containing compounds by utilization of benzyltriethylammonium tetrathiomolybdate $\left\{\left[\left(\mathrm{C}_{6} \mathrm{H}_{5} \mathrm{CH}_{2} \mathrm{NEt}_{3}\right)_{2^{-}}\right.\right.$ $\left.\left.\mathrm{MoS}_{4}\right] ; \mathbf{1}\right\}$ as a reagent, ${ }^{[19]}$ we have previously reported the synthesis of sugar disulfides with $\mathbf{1}$ as the key reagent. ${ }^{[20]}$ More recently, we have also reported the cleavage of disulfide bonds assisted by tetrathiomolybdate 1, which has resulted in the synthesis of a variety of sulfur-containing intermediates in a one-pot fashion. ${ }^{[21]}$ In this paper we extend the utility of tetrathiomolybdate $\mathbf{1}$ in carbohydrate chemistry, in an approach involving the formation of sugar disulfides, reductive cleavage of the disulfide bond, and subsequent conjugate addition of thiolates to suitable Michael acceptors in a one-pot domino reaction. Interestingly, this study has also resulted in the synthesis of a thiodisaccharide and a (thioglyco)amino acid, with good diastereoselectivity.

\section{Tandem Sulfur Transfer/Reduction/Michael Addition with Use of Benzyltriethylammonium Tetrathiomolybdate (1): Synthesis of Thioglycosides}

Glucose disulfide $\mathbf{5}^{[20]}$ was prepared by treatment of $\mathbf{1}$ with the glycosyl bromide 4 . In initial experiments, the glucose disulfide $\mathbf{5}$ was isolated and was then again treated 
with tetrathiomolybdate 1 ( 1 equiv., $28^{\circ} \mathrm{C}, 3 \mathrm{~h}$ ) in the presence of methyl vinyl ketone ( 2 equiv.) to furnish the thioglycoside $\mathbf{6}$ as the only product in 95\% yield (Scheme 1).

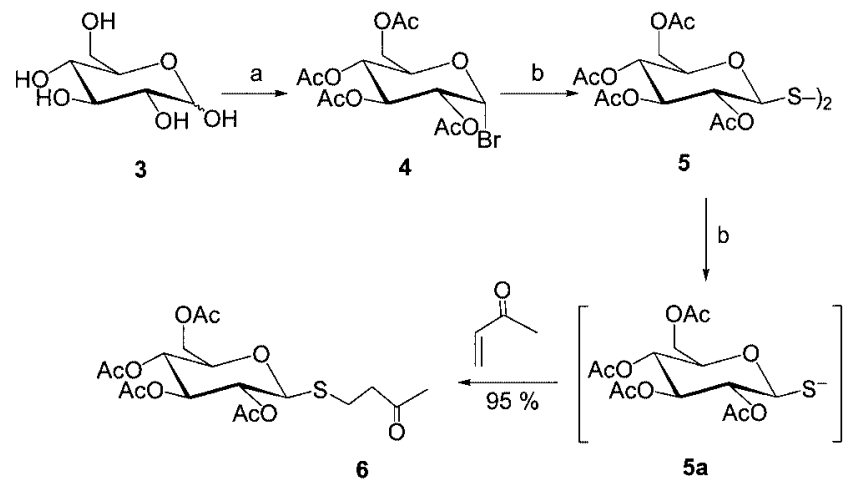

Scheme 1. Tandem sulfur transfer/reduction/Michael addition assisted by tetrathiomolybdate 1: a) i. $\left(\mathrm{CH}_{3} \mathrm{CO}\right)_{2} \mathrm{O}, \mathrm{HClO}_{4}$, ii. $30 \%$ $\mathrm{HBr}$ in acetic acid; b) $\mathrm{MoS}_{4}{ }^{2-}, \mathrm{CH}_{3} \mathrm{CN}$

In subsequent experiments, the reactions were carried out without isolation of the disulfide 5. Accordingly, treatment of glycosyl bromide 4 (1 equiv.) with tetrathiomolybdate $\mathbf{1}$ ( 2 equiv.) and acrylonitrile (1 equiv.) gave the corresponding thioglycoside 7 in very good yield $(90 \%)$. Similarly, treatment of glycosyl bromide $\mathbf{4}$ with tetrathiomolybdate $\mathbf{1}$ and methyl acrylate gave the thioglycoside $\mathbf{8}$ in excellent yield $(95 \%)$. Compounds 7 and $\mathbf{8}$ are good precursors for the synthesis of glycopeptides.

To ensure the generality of this methodology, the multistep, one-pot strategy was tested with other Michael acceptors such as acetamidomethyl acrylate and phenyl vinyl sulfone. Thus, sugar bromide 4 , on treatment with phenyl vinyl sulfone or acetamidomethyl acrylate in the presence of tetrathiomolybdate $\mathbf{1}$, afforded the corresponding thioglycoside $\mathbf{9}$ or (thioglyco)amino acid $\mathbf{1 0}$ (obtained as a 1:1 diastereomeric mixture), respectively, in good yields (Scheme 2).

\section{Tandem Sulfur Transfer/Reduction/Michael Addition Reaction with Ammonium Tetrathiomolybdate (2) in Aqueous Medium}

The usefulness of this reaction in aqueous medium is presented in Scheme 3. Thus, methyl 6-bromo-6-deoxy- $\alpha$-Dglucopyranoside (12), ${ }^{[22]}$ obtained from methyl $\alpha$-D-glucopyranoside 11 in a reaction with $\mathrm{PPh}_{3}$, and $\mathrm{CBr}_{4}$ in pyridine, was treated with ammonium tetrathiomolybdate (2) (2 equiv.) in the presence of methyl vinyl ketone in water as the solvent to give the Michael adduct $\mathbf{1 4}$ as the only product in good yield (82\%) (Scheme 3).

\section{An Intramolecular Version of Tandem Sulfur Transfer/ Reduction/Michael Addition Mediated by Tetrathiomolyb- date 1}

Encouraged by the results of the one-pot intermolecular disulfide formation/reduction/Michael addition sequence, we undertook an investigation of the intramolecular version

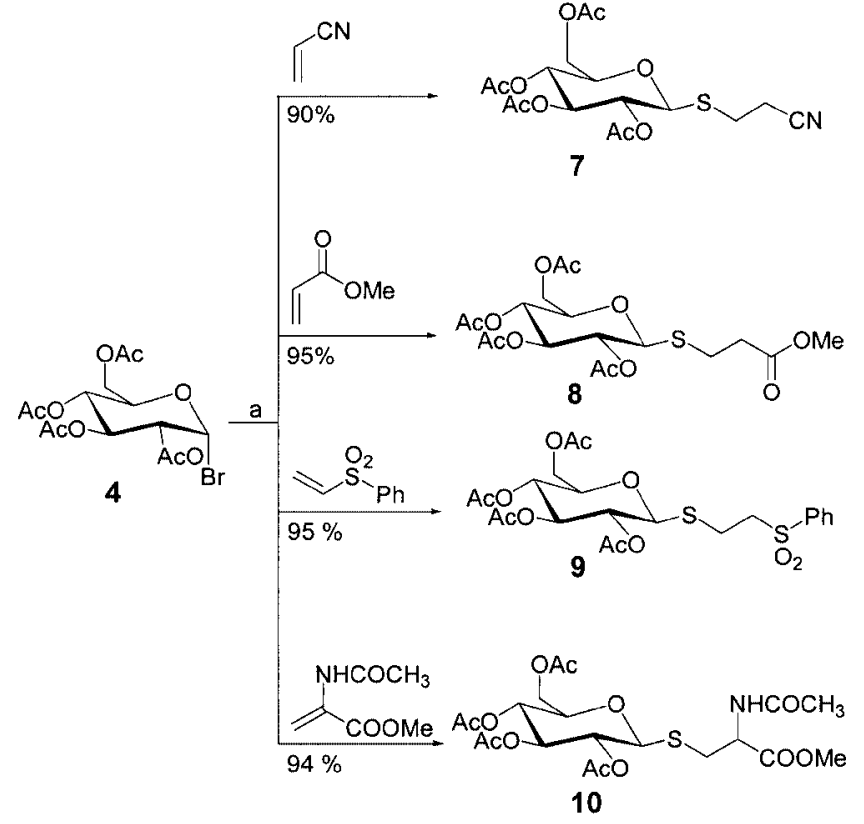

$1: 1$ diastereomers

Scheme 2. a) $\mathrm{MoS}_{4}{ }^{2-}$ (2 equiv.), $\mathrm{CH}_{3} \mathrm{CN}$

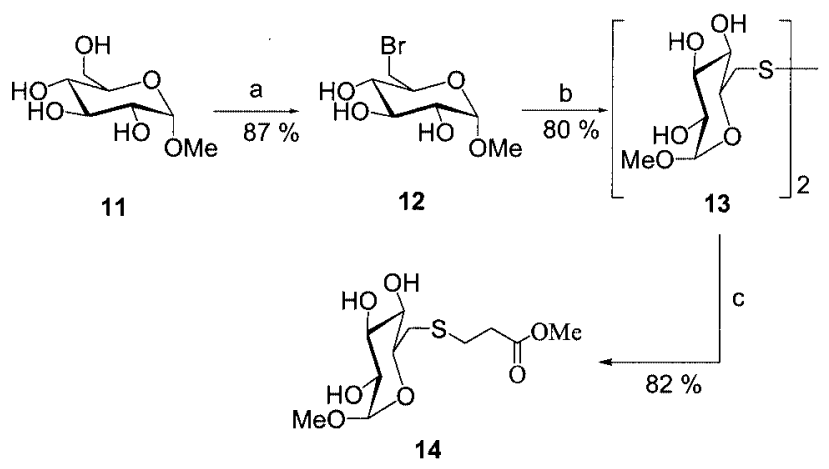

Scheme 3. Sulfur transfer/reduction/Michael addition in water: a) $\mathrm{PPh}_{3}, \mathrm{CBr}_{4}$, pyridine; b) $\left(\mathrm{NH}_{4}\right)_{2} \mathrm{MoS}_{4}, \mathrm{H}_{2} \mathrm{O}$; c) $\left(\mathrm{NH}_{4}\right)_{2} \mathrm{MoS}_{4}$, methyl acrylate, $\mathrm{H}_{2} \mathrm{O}$

of similar reactions with tetrathiomolybdate $\mathbf{1}$. The unsaturated lactone $\mathbf{1 6}$ was prepared from the glucal derivative $\mathbf{1 5}^{[23]}$ by treatment with $m \mathrm{CPBA}$ and $\mathrm{BF}_{3} \cdot \mathrm{OEt}_{2}$ at $0{ }^{\circ} \mathrm{C}$ in $\mathrm{CHCl}_{3}{ }^{[24]}$ Further treatment of compound $\mathbf{1 6}$ with $\mathbf{1}$ furnished the bicyclo[3.2.1] octane derivative $\mathbf{1 7}$ in $82 \%$ yield (Scheme 4). Likewise, treatment of $\mathbf{1 5}$ with $\mathrm{PhI}(\mathrm{OH}) \mathrm{OTs}$ in $\mathrm{CH}_{2} \mathrm{Cl}_{2}$ furnished the enone 18. This, on treatment with $\mathbf{1}$, afforded the bicyclo[3.2.1] octane derivative 19 in $65 \%$ yield. In an attempted synthesis of thiasugar derivatives by our multi-step, one-pot methodology, Michael acceptor $\mathbf{2 0}^{[25]}$ was treated with 1 under similar reaction conditions. However, the reaction gave only the disulfide $\mathbf{2 1}$, resulting from Michael addition of the hydroxy group on the unsaturated ester moiety followed by disulfide bond formation at the primary carbon center (Scheme 4). 

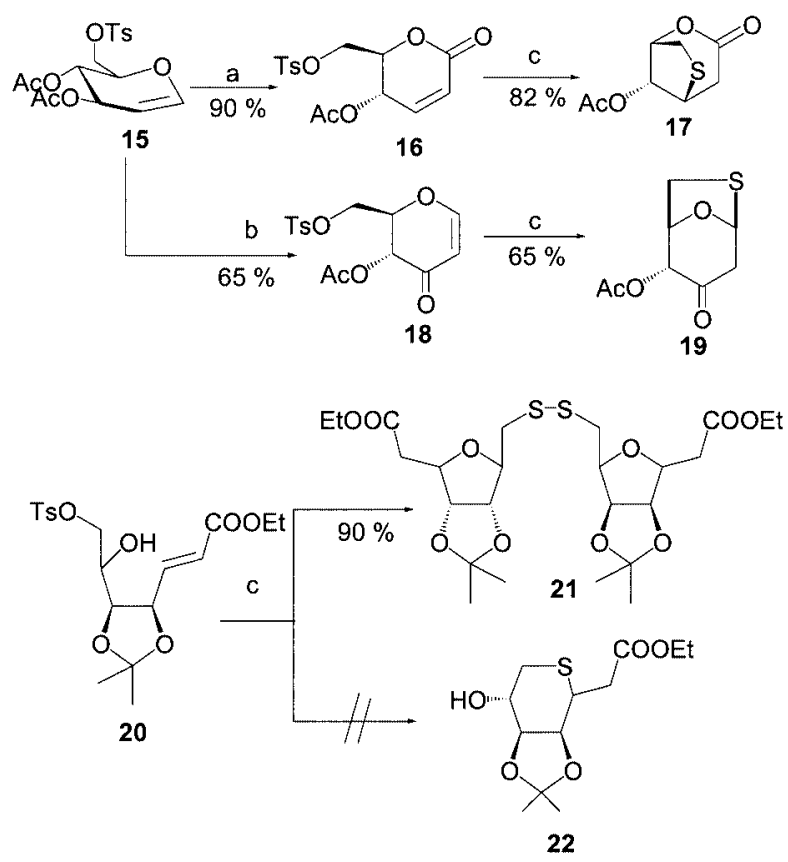

Scheme 4. An intramolecular version of the disulfide formation/ reduction/Michael addition sequence in one pot: (a) $m \mathrm{CPBA}$, $\mathrm{BF}_{3} \cdot \mathrm{Et}_{2} \mathrm{O}, \mathrm{CHCl}_{3}, 0{ }^{\circ} \mathrm{C}$; (b) $(\mathrm{PhI})(\mathrm{OH}) \mathrm{OTs}$, molecular sieves (4 A), $0-28{ }^{\circ} \mathrm{C}$; (c) $\mathrm{MoS}_{4}{ }^{2-}, \mathbf{1}, \mathrm{CH}_{3} \mathrm{CN}$

\section{Efforts towards Diastereoselective Michael Additions of Masked Thiolates Mediated by Benzyltriethylammonium Tetrathiomolybdate (1)}

To verify the diastereoselectivities of Michael addition of thiolates derived from sugar disulfides to $\alpha, \beta$-unsaturated carbonyl systems, we focused our attention on the reaction between tetrathiomolybdate $\mathbf{1}$ and sugar disulfide $\mathbf{5}$ in the presence of 2-cyclohexenone/2-cyclopentenone. The reactions furnished mixtures (1:1) of diastereomers 23/24, respectively, indicating that they are not diastereoselective (Scheme 5).

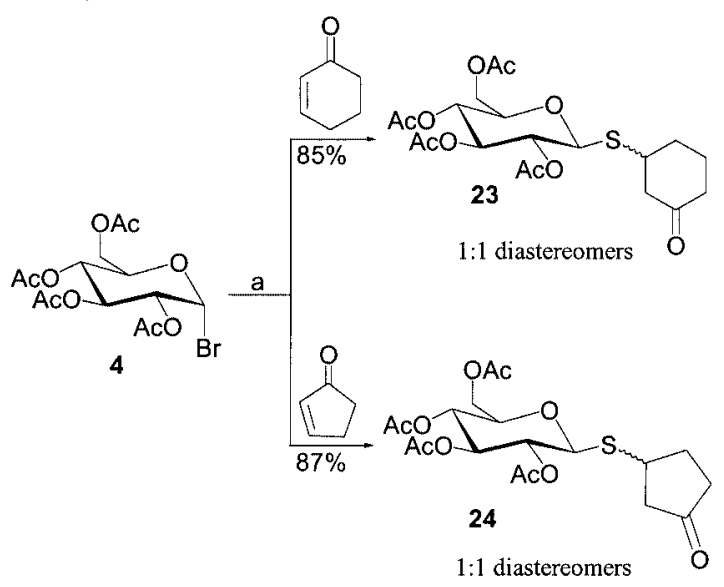

Scheme 5. One-pot disulfide formation/reduction/Michael addition sequence at a prochiral center: a) $\mathrm{MoS}_{4}{ }^{2-}, 1, \mathrm{CH}_{3} \mathrm{CN}$

However, the reaction between compound $\mathbf{2 5}^{[24]}$ and the dibenzyl disulfide $\mathbf{2 6}$ in the presence of $\mathbf{1}$ was highly diastereoselective, producing the Michael adduct 27 as the only product in excellent yield. The stereochemistry of 27 at $\mathrm{C}$ 3 was assigned from the NOE between the $3-\mathrm{H}$ equatorial and 4-H axial hydrogen atoms. The diastereoselective Michael addition was further demonstrated on treatment of sugar disulfide $\mathbf{2 8}^{[20]}$ with $\mathbf{2 5}$ in the presence of $\mathbf{1}$ to afford the sulfide 29, a thiodisaccharide, in excellent yield and with the axial isomer obtained as the only product. Similar treatment of 25 with Boc-Cys-OMe 30 in the presence of 1 produced a C-3-branched (thiogluco)amino acid derivative 31 in good yield (Scheme 6).

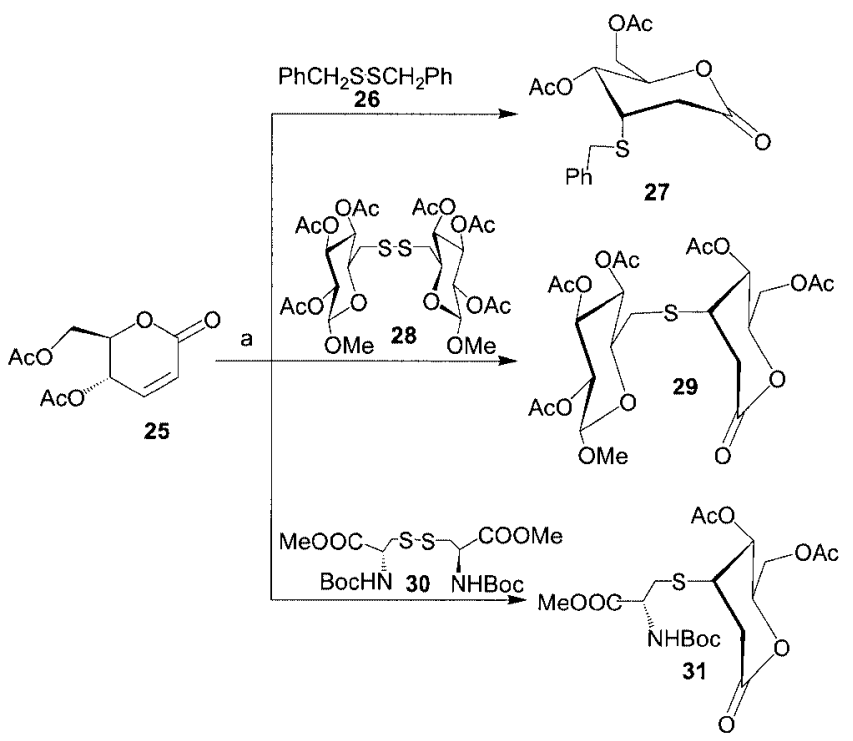

Scheme 6. Synthesis of 3-deoxy-3-thiosugar derivatives: a) $\mathrm{MoS}_{4}{ }^{2-}, \mathrm{CH}_{3} \mathrm{CN}$

\section{Conclusion}

Application of tetrathiomolybdate $\mathbf{1}$ in the synthesis of thiosaccharides by use of a sulfur transfer/reduction/ Michael addition methodology in a one-pot approach has been studied. An intramolecular version of this reaction for the synthesis of thiosugar analogues has also been studied. Finally, the synthesis of thiodisaccharide 29 and a C-3branched (thiogluco)amino acid $\mathbf{3 1}$ by the same methodology has been demonstrated.

\section{Experimental Section}

General Remarks: Molecular sieves were activated at $450{ }^{\circ} \mathrm{C}$ in a microwave oven for $10 \mathrm{~min}$. All the solvents were distilled from the appropriate drying agents. All the reactions were performed under anhydrous conditions and monitored by TLC on silica gel with UV indicator (Aldrich). Detection was by examination under UV light $(254 \mathrm{~nm})$ and by charring with $10 \%$ sulfuric acid in methanol. Column chromatography was performed on silica gel (Acme's, 100-200 mesh). Extracts were concentrated under reduced pressure at $<50{ }^{\circ} \mathrm{C}$ (bath). ${ }^{1} \mathrm{H}$ and ${ }^{13} \mathrm{C}$ NMR spectra were recorded with Bruker 300 and AMX 400 spectrometers. ${ }^{1} \mathrm{H}$ NMR spectra recorded in $\mathrm{CDCl}_{3}$ were referenced to residue $\mathrm{CHCl}_{3}$ at $\delta=$ $7.26 \mathrm{ppm}$, and ${ }^{13} \mathrm{C} \mathrm{NMR}$ spectra to the central peak of $\mathrm{CDCl}_{3}$ at 
$\delta=77.0 \mathrm{ppm}$. Mass spectra were recorded with a Shimadzu GCMS-QP5050 mass spectrometer.

Bis(2,3,4,6-tetra- $\boldsymbol{O}$-acetyl- $\boldsymbol{\beta}$-D-glucopyranosyl) Disulfide (5): ${ }^{[20]}$ Benzyltriethylammonium tetrathiomolybdate $(\mathbf{1}, \quad 0.89 \mathrm{~g}, \quad 1.47$ mmol) was added to a solution of 2,3,4,6-tetra- $O$-acetyl- $\alpha$-D-glucopyranosyl bromide $(4,0.55 \mathrm{~g}, 1.34 \mathrm{mmol})$ in $\mathrm{CH}_{3} \mathrm{CN}(6 \mathrm{~mL})$, and the reaction mixture was stirred at $0{ }^{\circ} \mathrm{C}$ for $24 \mathrm{~h}$. Most of the solvent was evaporated under reduced pressure, and the black material was extracted with $\mathrm{CH}_{2} \mathrm{Cl}_{2} / \mathrm{Et}_{2} \mathrm{O}(1: 4,15 \mathrm{~mL} \times 3)$. It was then filtered through a pad of Celite, and the same extraction was repeated four times. The combined filtrates, on removal of solvent, yielded the crude product, which on recrystallisation (ether/petroleum ether, 9:1) afforded the disulfide $5(0.462 \mathrm{~g}, 95 \%)$ as a white solid (m.p. $140-142{ }^{\circ} \mathrm{C}\left(\right.$ ref. $\left.^{[20]} 142-143{ }^{\circ} \mathrm{C}\right)$. IR (neat): $\tilde{v}=1720$, 2852, $2923 \mathrm{~cm}^{-1} .{ }^{1} \mathrm{H}$ NMR (300 MHz, $\left.\mathrm{CDCl}_{3}\right): \delta=2.00(\mathrm{~s}, 6 \mathrm{H})$, $2.03(\mathrm{~s}, 6 \mathrm{H}), 2.10(\mathrm{~s}, 6 \mathrm{H}), 2.13(\mathrm{~s}, 6 \mathrm{H}), 3.81\left(\mathrm{td}, J_{5,6 \mathrm{eq}}=1.8\right.$, $J_{4,5}=9.9 \mathrm{~Hz}, 2 \mathrm{H}$ ), 4.28 (pair of dd, $J_{5,6 \mathrm{eq}}=1.8, J_{5,6 \mathrm{ax}}=4.2$, $\left.J_{6 \mathrm{ax}, 6 \mathrm{eq}}=12.5 \mathrm{~Hz}, 4 \mathrm{H}\right), 4.9\left(\mathrm{~d}, J_{1,2}=9.4 \mathrm{~Hz}, 2 \mathrm{H}\right), 5.02-5.36(\mathrm{~m}$, $6 \mathrm{H}) \mathrm{ppm} .{ }^{13} \mathrm{C}$ NMR $\left(75 \mathrm{MHz}, \mathrm{CDCl}_{3}\right): \delta=20.1,61.2,67.4,69.2$, 73.4, 75.6, 86.6, 168.9, 169.5, 170.2 ppm. Low-resolution MS: calcd: $m / z 726\left[\mathrm{M}^{+}\right]$, found $727\left[\mathrm{M}^{+}+1\right] . \mathrm{C}_{28} \mathrm{H}_{38} \mathrm{O}_{18} \mathrm{~S}_{2}$ (726.7): calcd. C 46.28, H 5.23; found C 45.95, H 5.25.

Typical Experimental Procedure for the One-Pot Tandem Sulfur Transfer/Reduction/Michael Addition: Tetrathiomolybdate $\mathbf{1}$ $(0.609 \mathrm{~g}, 1 \mathrm{mmol})$ was added in one portion to a well-stirred solution of sugar bromide $(1 \mathrm{mmol})$ in $\mathrm{CH}_{3} \mathrm{CN}(10 \mathrm{~mL})$, and the reaction mixture was stirred at $28^{\circ} \mathrm{C}$ for $2 \mathrm{~h}$. The reaction was monitored by TLC. Once the starting material had disappeared (30 min), tetrathiomolybdate $\mathbf{1}(0.609 \mathrm{~g}, 1 \mathrm{mmol})$ was once again added, followed by addition of the Michael acceptor $(1 \mathrm{mmol})$ and the reaction mixture was stirred under argon at room temperature for $3 \mathrm{~h}$. The solvent was removed under vacuum. The black residue was extracted with diethyl ether/dichloromethane mixture $(4: 1,25 \mathrm{~mL}$ $\times 4)$ and filtered through a Celite pad. The filtrate was concentrated under vacuum and the crude product was purified by flash column chromatography on silica gel.

4-(2,3,4,6-Tetra- $\boldsymbol{O}$-acetyl- $\boldsymbol{\beta}$-D-glucopyranosylthio)butan-2-one (6): IR (neat): $\left.\tilde{v}=1748,2940 \mathrm{~cm}^{-1} .{ }^{1} \mathrm{H} \mathrm{NMR} \mathrm{(300} \mathrm{MHz,} \mathrm{CDCl}_{3}\right): \delta=$ $2.01(\mathrm{~s}, 3 \mathrm{H}), 2.03(\mathrm{~s}, 3 \mathrm{H}), 2.05(\mathrm{~s}, 3 \mathrm{H}), 2.09(\mathrm{~s}, 3 \mathrm{H}), 2.17$ (s, 3 $\mathrm{H}), 2.75-2.98(\mathrm{~m}, 4 \mathrm{H}), 3.67-3.73(\mathrm{~m}, 1 \mathrm{H}), 4.15(\mathrm{dd}, J=2.1$, $J=12.3 \mathrm{~Hz}, 1 \mathrm{H}), 4.23(\mathrm{dd}, J=4.8, J=12.3 \mathrm{~Hz}, 1 \mathrm{H}), 4.52(\mathrm{~d}$, $J=9.9 \mathrm{~Hz}, 1 \mathrm{H}), 5.20(\mathrm{t}, J=9.6 \mathrm{~Hz}, 1 \mathrm{H}), 5.08(\mathrm{t}, J=9.9 \mathrm{~Hz}, 1$ $\mathrm{H}), 5.22(\mathrm{t}, J=9 \mathrm{~Hz}, 1 \mathrm{H}) \mathrm{ppm} .{ }^{13} \mathrm{C} \mathrm{NMR}\left(75 \mathrm{MHz}, \mathrm{CDCl}_{3}\right): \delta=$ 20.5, 20.6, 20.7, 23.8, 30.0, 44.1, 62.0, 68.2, 69.7, 73.8, 75.9, 83.9, 169.4, 169.4, 170.1, 170.6, 206.4 ppm. Low-resolution MS: calcd: $m / z 434\left[\mathrm{M}^{+}\right]$, found $435\left[\mathrm{M}^{+}+1\right] . \mathrm{C}_{18} \mathrm{H}_{26} \mathrm{O}_{10} \mathrm{~S}$ (434.5): calcd. $\mathrm{C}$ 49.76, H 6.03; found C 49.41, H 5.78.

3-(2,3,4,6-Tetra- $O$-acetyl- $\beta$-D-glucopyranosylthio)propionitrile (7): $[\alpha]_{\mathrm{D}}^{25}=-32(c=1$, chloroform). IR (neat): $\tilde{v}=1752,2250,2853$, $2925 \mathrm{~cm}^{-1} .{ }^{1} \mathrm{H}$ NMR $\left(300 \mathrm{MHz}, \mathrm{CDCl}_{3}\right): \delta=1.98(\mathrm{~s}, 3 \mathrm{H}), 2.00$ (s, $3 \mathrm{H}), 2.03$ (s, $3 \mathrm{H}), 2.07$ (s, $3 \mathrm{H}), 2.63-2.77$ (m, $2 \mathrm{H}), 2.86-2.88$ $(\mathrm{m}, 1 \mathrm{H}), 2.96-3.05(\mathrm{~m}, 1 \mathrm{H}), 3.69-3.74(\mathrm{~m}, 1 \mathrm{H}), 4.16(\mathrm{dd}, J=$ $2.1, J=12.3 \mathrm{~Hz}, 1 \mathrm{H}), 4.20(\mathrm{dd}, J=4.8, J=12.3 \mathrm{~Hz}, 1 \mathrm{H}), 4.55$ $(\mathrm{d}, J=10.2 \mathrm{~Hz}, 1 \mathrm{H}), 5.00(\mathrm{t}, J=9.3 \mathrm{~Hz}, 1 \mathrm{H}), 5.04(\mathrm{t}, J=$ $10.2 \mathrm{~Hz}, 1 \mathrm{H}), 5.20(\mathrm{t}, J=9.3 \mathrm{~Hz}, 1 \mathrm{H}) \mathrm{ppm} .{ }^{13} \mathrm{C} \mathrm{NMR}(75 \mathrm{MHz}$, $\left.\mathrm{CDCl}_{3}\right): \delta=19.6,20.5,20.6,20.7,25.7,61.9,68.0,69.4,73.5,76.1$, $83.3,118.0,169.3,169.4,170.0,170.5$ ppm. Low-resolution MS: calcd. $m / z 417\left[\mathrm{M}^{+}\right]$, found $418\left[\mathrm{M}^{+}+1\right] . \mathrm{C}_{17} \mathrm{H}_{23} \mathrm{NO}_{9} \mathrm{~S}$ (417.4): calcd. C 48.91, H 5.55; found C 49.26, H 5.65.

Methyl 3-(2,3,4,6-Tetra- $\boldsymbol{O}$-acetyl- $\beta$-D-glucopyranosylthio)propionate (8): IR (neat): $\tilde{v}=1750,2852,2923 \mathrm{~cm}^{-1} .{ }^{1} \mathrm{H}$ NMR (300 MHz,
$\left.\mathrm{CDCl}_{3}\right): \delta=2.01(\mathrm{~s}, 3 \mathrm{H}), 2.03(\mathrm{~s}, 3 \mathrm{H}), 2.05(\mathrm{~s}, 3 \mathrm{H}), 2.09(\mathrm{~s}, 3 \mathrm{H})$, $2.68(\mathrm{t}, J=6.9 \mathrm{~Hz}, 2 \mathrm{H}), 2.83-3.04(\mathrm{~m}, 2 \mathrm{H}), 3.69(\mathrm{~s}, 3 \mathrm{H})$, $3.72-3.74(\mathrm{~m}, 1 \mathrm{H}), 4.14(\mathrm{dd}, J=2.1, J=12.3 \mathrm{~Hz}, 1 \mathrm{H}), 4.23(\mathrm{dd}$, $J=4.8, J=12.6 \mathrm{~Hz}, 1 \mathrm{H}), 4.54(\mathrm{~d}, J=9.9 \mathrm{~Hz}, 1 \mathrm{H}), 5.02(\mathrm{t}, J=$ $9.3 \mathrm{~Hz}, 1 \mathrm{H}), 5.08(\mathrm{t}, J=9.3 \mathrm{~Hz}, 1 \mathrm{H}), 5.22(\mathrm{t}, J=9.3 \mathrm{~Hz}, 1 \mathrm{H})$ ppm. ${ }^{13} \mathrm{C} \mathrm{NMR}\left(75 \mathrm{MHz}, \mathrm{CDCl}_{3}\right): \delta=20.5,20.6,25.3,35.2,51.8$, $62.1,68.3,69.7,73.7,75.9,83.9,169.3,169.4,170.1,170.6,172.0$ ppm. Low-resolution MS: calcd. $m / z 450\left[\mathrm{M}^{+}\right]$, found $473\left[\mathrm{M}^{+}+\right.$ Na]. $\mathrm{C}_{18} \mathrm{H}_{26} \mathrm{O}_{11} \mathrm{~S}$ (450.5): calcd. C 47.99, H 5.82; found C 48.36, H 5.88 .

Phenyl 2-(2,3,4,6-Tetra- $O$-acetyl- $\beta$-D-glucopyranosylthio)ethyl Sulfone (9): $[\alpha]_{\mathrm{D}}^{25}=-33(c=1$, chloroform). IR (neat): $\tilde{v}=1227$, $1633,1748,2946 \mathrm{~cm}^{-1} .{ }^{1} \mathrm{H}$ NMR $\left(300 \mathrm{MHz}, \mathrm{CDCl}_{3}\right): \delta=2.00(\mathrm{~s}$, $3 \mathrm{H}), 2.02(\mathrm{~s}, 3 \mathrm{H}), 2.03(\mathrm{~s}, 3 \mathrm{H}), 2.13(\mathrm{~s}, 3 \mathrm{H}), 2.80-2.90$ (m, 1 H), 2.95-3.05 (m, $1 \mathrm{H}), 3.38-3.48(\mathrm{~m}, 2 \mathrm{H}), 3.67-3.71(\mathrm{~m}, 1 \mathrm{H})$, $4.10-4.16(\mathrm{~m}, 2 \mathrm{H}), 4.48(\mathrm{~d}, J=9.9 \mathrm{~Hz}, 1 \mathrm{H}), 4.89(\mathrm{t}, J=9 \mathrm{~Hz}$, $1 \mathrm{H}), 5.00(\mathrm{t}, J=9.6 \mathrm{~Hz}, 1 \mathrm{H}), 5.19(\mathrm{t}, J=8.4 \mathrm{~Hz}, 1 \mathrm{H}), 7.61(\mathrm{t}$, $J=6.9 \mathrm{~Hz}, 2 \mathrm{H}), 7.69(\mathrm{~d}, J=6.9 \mathrm{~Hz}, 1 \mathrm{H}), 7.93(\mathrm{~d}, J=8.1 \mathrm{~Hz}, 2$ H) ppm. ${ }^{13} \mathrm{C}$ NMR $\left(75 \mathrm{MHz}, \mathrm{CDCl}_{3}\right): \delta=20.3,20.4,20.5,20.6$, 23.0, 56.9, 61.8, 67.9, 69.2, 73.3, 75.9, 86.5, 128.0, 129.4, 134.0, 138.4, 169.2, 169.3, 169.9, 170.6 ppm. Low-resolution MS: calcd. $\mathrm{m} / \mathrm{z} 532\left[\mathrm{M}^{+}\right]$, found $533\left[\mathrm{M}^{+}+1\right] . \mathrm{C}_{22} \mathrm{H}_{28} \mathrm{O}_{11} \mathrm{~S}_{2}$ (532.6): calcd. $\mathrm{C}$ 49.61, H 5.30; found C 49.83, H 5.22.

$\mathrm{N}$-Acetyl-3-(2,3,4,6-tetra- $O$-acetyl- $\beta$-D-glucopyranosylthio)-DLcysteine Methyl Ester (10): ${ }^{1} \mathrm{H}$ NMR $\left(300 \mathrm{MHz}, \mathrm{CDCl}_{3}\right): \delta=$ 1.95-2.06 (m, $15 \mathrm{H}), 2.94-3.03(\mathrm{~m}, 1 \mathrm{H}), 3.10-3.18(\mathrm{~m}, 1 \mathrm{H})$, $3.70(\mathrm{~s}, 3 \mathrm{H}), 3.95-4.15(\mathrm{~m}, 3 \mathrm{H}), 4.84(\mathrm{t}, J=9.6 \mathrm{~Hz}, 1 \mathrm{H})$, $4.72-4.78(\mathrm{~m}, 1 \mathrm{H}), 4.89-5.01(\mathrm{~m}, 2 \mathrm{H}), 5.16(\mathrm{dt}, J=2.7, J=$ $9.3 \mathrm{~Hz}, 1 \mathrm{H}), 6.55(\mathrm{~d}, J=7.2 \mathrm{~Hz}, 1 \mathrm{H}), 6.62(\mathrm{~d}, J=7.2 \mathrm{~Hz}, 1 \mathrm{H})$ ppm. ${ }^{13} \mathrm{C} \mathrm{NMR}\left(75 \mathrm{MHz}, \mathrm{CDCl}_{3}\right): 14.1,20.4,20.5,20.9,22.7,31.5$, $32.2,51.7,52.2,52.5,52.6,60.3,61.7,62.0,67.9,68.0,69.6,69.7$, 73.4, 75.8, 76.0, 83.1, 83.5, 169.2, 169.3, 169.4, 169.8, 169.9, 170.5, 170.6, $170.8 \mathrm{ppm}$. Low-resolution MS: calcd. $\mathrm{m} / z 507\left[\mathrm{M}^{+}\right]$, found $530\left[\mathrm{M}^{+}+\mathrm{Na}\right]$. High-resolution MS: calcd. for $\mathrm{C}_{20} \mathrm{H}_{29} \mathrm{NO}_{12} \mathrm{~S}+$ $\mathrm{Na} 530.1409$; found 530.1414.

Methyl 3-(6-Deoxy- $\alpha$-D-glucopyranos-6-ylthio)propionate (14): Ammonium tetrathiomolybdate $(2,0.262 \mathrm{~g}, 1 \mathrm{mmol})$ was added to a solution of methyl 6-bromo-6-deoxy- $\alpha$-D-glucopyranoside (12, $0.256 \mathrm{~g}, 1 \mathrm{mmol})$ in water $(6 \mathrm{~mL})$, and the reaction mixture was stirred for $4 \mathrm{~h}$. The reaction was monitored by TLC. Once the starting material had disappeared $(3 \mathrm{~h})$, ammonium tetrathiomolybdate $(2,0.262 \mathrm{~g}, 1 \mathrm{mmol})$ was once again added, followed by methyl acrylate $(0.086 \mathrm{~g}, 1 \mathrm{mmol})$, and the reaction mixture was stirred at room temperature for a further $3 \mathrm{~h}$. The solvent was removed under vacuum. The black residue was extracted with methanol $(10 \times$ $3 \mathrm{~mL}$ ) and filtered through a Celite pad. The combined filtrates were concentrated under vacuum and the crude product was purified by flash column chromatography on silica gel (eluent: methanol/chloroform, 1:9) to yield compound $14(0.243 \mathrm{~g}, 82 \%)$ as a colorless, gummy solid. ${ }^{1} \mathrm{H}$ NMR $\left(300 \mathrm{MHz}, \mathrm{CDCl}_{3}\right): \delta=2.65(\mathrm{t}$, $J=7.2 \mathrm{~Hz}, 2 \mathrm{H}), 2.71-2.76(\mathrm{~m}, 1 \mathrm{H}), 2.88(\mathrm{t}, J=7.2 \mathrm{~Hz}, 2 \mathrm{H})$, $3.10(\mathrm{~m}, 1 \mathrm{H}), 3.36(\mathrm{t}, J=9 \mathrm{~Hz}, 1 \mathrm{H}), 3.44(\mathrm{~s}, 3 \mathrm{H}), 3.53(\mathrm{dd}, J=$ $3.3, J=9.6 \mathrm{~Hz}, 1 \mathrm{H}), 3.70(\mathrm{~m}, 5 \mathrm{H}), 4.73(\mathrm{~d}, J=3.3 \mathrm{~Hz}, 1 \mathrm{H})$ ppm. ${ }^{13} \mathrm{C} \mathrm{NMR}\left(75 \mathrm{MHz}, \mathrm{CDCl}_{3}\right): \delta=28.1,33.7,34.6,51.8,55.2$, 71.6, 72.0, 72.7, 74.1, 99.2, 172.6 ppm. Low-resolution MS: calcd. $\mathrm{m} / z 296\left[\mathrm{M}^{+}\right]$, found $297\left[\mathrm{M}^{+}+1\right]$. High-resolution MS: calcd. for $\mathrm{C}_{11} \mathrm{H}_{20} \mathrm{O}_{7} \mathrm{~S}+\mathrm{Na} 319.0828$; found 319.0822.

$\boldsymbol{\alpha}, \boldsymbol{\beta}$-Unsaturated $\boldsymbol{\delta}$-Lactone 16: ${ }^{[24]} 2,3$-Di- $O$-acetyl-6- $O$-tosyl-D-glucal $(15,0.3 \mathrm{~g}, 0.78 \mathrm{mmol})$ was placed in a $50-\mathrm{mL}$ round-bottomed flask and cooled to $-20{ }^{\circ} \mathrm{C}$ with the aid of an ice/sodium chloride mixture. $m$-Chloroperbenzoic acid $(0.163 \mathrm{~g}, 0.93 \mathrm{mmol})$ was dis- 
solved in dry chloroform $(3 \mathrm{~mL})$ and added dropwise to the reaction mixture over a period of $10 \mathrm{~min} . \mathrm{BF}_{3} \cdot \mathrm{Et}_{2} \mathrm{O}(66 \mu \mathrm{L}, 0.67 \mathrm{mmol})$ was added to this mixture, and stirring was continued for $1 \mathrm{~h}$. The reaction mixture was quenched with ammonia solution $(0.3 \mathrm{~mL})$ and ice-cold water $(6.5 \mathrm{~mL})$. After the mixture had been stirred for $10 \mathrm{~min}$, the organic layer was separated. The aqueous layer was extracted twice with chloroform $(10 \mathrm{~mL})$. The combined chloroform layers were washed with water $(10 \mathrm{~mL})$, ammonia solution $(10 \mathrm{~mL})$, again with water $(10 \mathrm{~mL})$, and with brine solution $(10 \mathrm{~mL})$. The organic layer was dried with anhydrous sodium sulfate and filtered, and the solvent was removed to obtain the crude product, which was purified by flash column chromatography (ethyl acetate/petroleum ether, 1:3) to provide pure compound $\mathbf{1 6}$ $(0.199 \mathrm{~g}, 75 \%)$ as a colorless solid. M.p. $93-94{ }^{\circ} \mathrm{C}$ (ref. ${ }^{[24]} 92-93$ ${ }^{\circ} \mathrm{C}$ ) (the product is unstable at room temperature and decomposes to a black gum in one week). IR (neat): $\tilde{v}=1744 \mathrm{~cm}^{-1} \cdot{ }^{1} \mathrm{H}$ NMR $\left(300 \mathrm{MHz}, \mathrm{CDCl}_{3}\right): \delta=2.12(\mathrm{~s}, 3 \mathrm{H}), 2.46(\mathrm{~s}, 3 \mathrm{H}), 4.24$ (t, $J_{5,6}=$ $3.6 \mathrm{~Hz}, 2 \mathrm{H}), 4.65-4.61(\mathrm{~m}, 1 \mathrm{H}), 5.53\left(\mathrm{~d}, J_{4,5}=6.6 \mathrm{~Hz}, 1 \mathrm{H}\right), 6.07$ $\left(\mathrm{d}, J_{2,3}=9.9 \mathrm{~Hz}, 1 \mathrm{H}\right), 6.78\left(\mathrm{dd}, J_{3,4}=3, J_{2,3}=9.9 \mathrm{~Hz}, 1 \mathrm{H}\right), 7.37$ $(\mathrm{d}, J=8.1 \mathrm{~Hz}, 2 \mathrm{H}), 7.79(\mathrm{~d}, J=8.1 \mathrm{~Hz}, 2 \mathrm{H}) \mathrm{ppm} .{ }^{13} \mathrm{C} \mathrm{NMR}$ $\left(75 \mathrm{MHz}, \mathrm{CDCl}_{3}\right): \delta=20.6,21.6,63.3,66.6,76.7,122.0,130.0$, $128.0,131.9,143.3,145.4,160.5,169.5 \mathrm{ppm}$.

3-Oxo-2-oxa-6-thiabicyclo[3.2.1]oct-8-yl Acetate (17): The tetrathiomolybdate $1(0.347 \mathrm{~g}, 0.57 \mathrm{mmol})$ was added to a solution of $\delta$-lactone $16(0.1 \mathrm{~g}, 0.285 \mathrm{mmol})$ in acetonitrile $(5 \mathrm{~mL})$, and the system was stirred at room temperature for $12 \mathrm{~h}$. The solvent was evaporated under reduced pressure, and the black residue was extracted with dichloromethane and diethyl ether $(1: 5,25 \mathrm{~mL} \times 5)$, filtered through a Celite pad, and concentrated. The residue was purified by column chromatography on silica gel (eluent: ethyl acetate/petroleum ether, 1:1) to afford the bicyclic lactone $17(0.047 \mathrm{~g}$, $82 \%$ ). IR (neat): $\tilde{v}=1730,1745,2853,2923 \mathrm{~cm}^{-1} .{ }^{1} \mathrm{H}$ NMR $\left(300 \mathrm{MHz}, \mathrm{CDCl}_{3}\right): \delta=2.16(\mathrm{~s}, 3 \mathrm{H}), 2.88(\mathrm{~d}, J=18.9 \mathrm{~Hz}, 1 \mathrm{H})$, $3.11(\mathrm{~d}, J=4.2 \mathrm{~Hz}, 1 \mathrm{H}), 3.17(\mathrm{~d}, J=4.2 \mathrm{~Hz}, 1 \mathrm{H}), 3.26(\mathrm{~d}, J=$ $12 \mathrm{~Hz}, 1 \mathrm{H}), 3.56(\mathrm{~m}, 1 \mathrm{H}), 5.08$ (br. s, $1 \mathrm{H}) 5.16(\mathrm{~m}, 1 \mathrm{H}) \mathrm{ppm}$. ${ }^{13} \mathrm{C}$ NMR $\left(75 \mathrm{MHz}, \mathrm{CDCl}_{3}\right): \delta=20.7,32.8,37.5,37.7,72.3,78.8$, 167.2, 169.9 ppm. Low-resolution MS: calcd. $m / z 202\left[\mathrm{M}^{+}\right]$, found $202\left[\mathrm{M}^{+}\right]$. High-resolution MS: calcd. for $\mathrm{C}_{8} \mathrm{H}_{10} \mathrm{O}_{4} \mathrm{~S}+\mathrm{Na}$ 225.0198; found 225.0195 .

(+)-(2R,3R)-3-(Acetoxy)-2-(p-tolylsulfonyloxymethyl)-2,3-dihydro4H-pyran-4-one (18): A suspension of 3,4-di- $O$-acetyl-6- $O$-tosyl-Dglucal $(\mathbf{1 5}, 0.1 \mathrm{~g}, 0.26 \mathrm{mmol})$ and powdered molecular sieves $(4 \AA$, $0.025 \mathrm{~g})$ in dry acetonitrile $(4 \mathrm{~mL})$ was stirred under nitrogen at 0 ${ }^{\circ} \mathrm{C}$ for $5 \mathrm{~min}$. [Hydroxy(tosyloxy)iodo]benzene ( $\left.0.122 \mathrm{~g}, 0.31 \mathrm{mmol}\right)$ was added in one portion, and the temperature was raised to room temperature. During the following $30 \mathrm{~min}$ the suspension turned yellow and then back to a colorless form. After $75 \mathrm{~min}$, the suspension was filtered through a pad of Celite and the residue was washed with dichloromethane. The combined filtrates were washed with aqueous $\mathrm{NaHCO}_{3}(5 \mathrm{~mL})$ and brine $(5 \mathrm{~mL})$, dried $\left(\mathrm{MgSO}_{4}\right)$, and concentrated under reduced pressure to give a yellow oil. The crude product was rapidly purified by flash column chromatography on silica gel (eluent: ethyl acetate/petroleum ether, 3:7) to provide the enone $18(0.55 \mathrm{~g}, 45 \%)$ as a colorless gum. IR (neat): $\tilde{v}=$ 1671, 1710, 1745, $2954 \mathrm{~cm}^{-1} .{ }^{1} \mathrm{H}$ NMR $\left(300 \mathrm{MHz}, \mathrm{CDCl}_{3}\right): \delta=$ $2.11(\mathrm{~s}, 3 \mathrm{H}), 2.46(\mathrm{~s}, 3 \mathrm{H}), 4.27(\mathrm{dd}, J=4.5, J=11.1 \mathrm{~Hz}, 1 \mathrm{H})$, $4.39(\mathrm{dd}, J=1.8, J=11.1 \mathrm{~Hz}, 1 \mathrm{H}), 4.58(\mathrm{~m}, 1 \mathrm{H}), 5.44(\mathrm{~d}, J=$ $2.4 \mathrm{~Hz}, 1 \mathrm{H}), 5.47(\mathrm{~d}, J=4.5 \mathrm{~Hz}, 1 \mathrm{H}), 7.27(\mathrm{~d}, J=6.6 \mathrm{~Hz}, 1 \mathrm{H})$, $7.37(\mathrm{~d}, J=8.1 \mathrm{~Hz}, 2 \mathrm{H}), 7.80(\mathrm{~d}, J=8.1 \mathrm{~Hz}, 2 \mathrm{H}) \mathrm{ppm} .{ }^{13} \mathrm{C}$ NMR (75 MHz, $\mathrm{CDCl}_{3}$ ): $\delta=20.3,21.7,66.2,67.8,77.5,105.4$, 128.1, 130.0, 132.2, 145.4, 162.0, 168.8, 187.3 ppm. Low-resolution MS: calcd. $m / z 340\left[\mathrm{M}^{+}\right]$, found $340\left[\mathrm{M}^{+}\right]$. High-resolution MS: calcd. for $\mathrm{C}_{15} \mathrm{H}_{16} \mathrm{O}_{7} \mathrm{~S}+\mathrm{Na}$ 363.0616; found 363.0618.
3-Oxo-8-oxa-6-thiabicyclo[3.2.1]oct-2-yl Acetate (19): Tetrathiomolybdate $1(0.87 \mathrm{~g}, 0.142 \mathrm{mmol})$ was added to a solution of $4-O$ acetyl-1,5-anhydro-2-deoxy-6-O-tosyl-D-erythro-hex-1-en-3-ulose $(18,0.55 \mathrm{~g}, 0.119 \mathrm{mmol})$ in acetonitrile $(5 \mathrm{~mL})$, and the reaction mixture was stirred at room temperature for $12 \mathrm{~h}$. The solvent was evaporated under reduced pressure, the black residue was extracted with dichloromethane and diethyl ether $(2: 10,12 \mathrm{~mL} \times 5)$ and filtered through a Celite pad, and the filtrate was concentrated. The crude product was purified by flash column chromatography on silica gel (eluent: ethyl acetate/petroleum ether, 1:1) to afford the acetate $19(0.013 \mathrm{~g}, 55 \%)$ as a pale yellow oil. IR (neat): $\tilde{v}=1730$, 1745, $2958 \mathrm{~cm}^{-1}$. ${ }^{1} \mathrm{H}$ NMR (300 MHz, $\left.\mathrm{CDCl}_{3}\right): \delta=2.15(\mathrm{~s}, 3 \mathrm{H})$, $2.69(\mathrm{~d}, J=15.9 \mathrm{~Hz}, 1 \mathrm{H}), 4.80$ (br. s, $1 \mathrm{H}), 3.10-3.27$ (m, $3 \mathrm{H})$, $5.08(\mathrm{~d}, J=6.6 \mathrm{~Hz}, 1 \mathrm{H}), 5.83(\mathrm{~m}, 1 \mathrm{H}) \mathrm{ppm} .{ }^{13} \mathrm{C} \mathrm{NMR}(75 \mathrm{MHz}$, $\left.\mathrm{CDCl}_{3}\right): \delta=20.6,34.2,50.1,76.1,80.9,81.1,169.5,198.2 \mathrm{ppm}$. Low-resolution MS: calcd. $m / z 202\left[\mathrm{M}^{+}\right]$, found $202\left[\mathrm{M}^{+}\right]$. Highresolution MS: calcd. for $\mathrm{C}_{8} \mathrm{H}_{10} \mathrm{O}_{4} \mathrm{~S}+\mathrm{Na}$ 225.0198; found 225.0199 .

Treatment of Hydroxy Ester 20 with Tetrathiomolybdate 1. Synthesis of Compound 21: Tetrathiomolybdate 1 ( $2.15 \mathrm{~g}, 3.53 \mathrm{mmol})$ was added to a solution of compound $20(0.69 \mathrm{~g}, 1.73 \mathrm{mmol})$ in acetonitrile $(10 \mathrm{~mL})$, and the mixture was stirred at room temperature for $12 \mathrm{~h}$. The solvent was evaporated under reduced pressure, the black residue was extracted with dichloromethane and diethyl ether $(3: 10,25 \mathrm{~mL} \times 4)$ and filtered through a Celite pad, and the filtrate was concentrated. The residue was purified by flash column chromatography (eluent: ethyl acetate/petroleum ether, 3:7) to afford $21(0.423 \mathrm{~g}, 90 \%)$ as a gummy solid. IR (neat): $\tilde{v}=1743,2943$, $2985 \mathrm{~cm}^{-1} .{ }^{1} \mathrm{H}$ NMR $\left(300 \mathrm{MHz}, \mathrm{CDCl}_{3}\right): \delta=1.26(\mathrm{t}, J=6.6 \mathrm{~Hz}$, $3 \mathrm{H}), 1.35(\mathrm{~s}, 3 \mathrm{H}), 1.54(\mathrm{~s}, 3 \mathrm{H}), 2.64(\mathrm{dd}, J=3.3, J=5.4 \mathrm{~Hz}, 2$ H), $2.98(\mathrm{~d}, J=6.3 \mathrm{~Hz}, 2 \mathrm{H}), 4.10-4.29(\mathrm{~m}, 4 \mathrm{H}), 4.53-4.59(\mathrm{~m}$, $2 \mathrm{H}) \mathrm{ppm} .{ }^{13} \mathrm{C} \mathrm{NMR}\left(75 \mathrm{MHz}, \mathrm{CDCl}_{3}\right): \delta=14.1,25.4,27.2,38.2$, 42.3, 60.6, 80.5, 82.8, 83.7, 84.2, 114.7, 170.2 ppm. Low-resolution MS: calcd. $m / z 550\left[\mathrm{M}^{+}\right]$, found $573[\mathrm{M}+\mathrm{Na}$. High-resolution MS: calcd. for $\mathrm{C}_{24} \mathrm{H}_{38} \mathrm{O}_{4} \mathrm{~S}_{2}+\mathrm{Na} 573.1905$; found 573.1907.

3-(2,3,4,6-Tetra- $O$-acetyl- $\beta$-D-glucopyranosylthio)cyclohexanone (23): $[\alpha]_{\mathrm{D}}^{25}=-24$ ( $c=1$, chloroform). IR (neat): $\tilde{v}=1713,1752$, 2854, $2926 \mathrm{~cm}^{-1}$. ${ }^{1} \mathrm{H}$ NMR $\left(300 \mathrm{MHz}, \mathrm{CDCl}_{3}\right): \delta=1.63-1.81(\mathrm{~m}$, $2 \mathrm{H}), 2.01$ (s, $3 \mathrm{H}), 2.03$ (s, $3 \mathrm{H}), 2.05(\mathrm{~s}, 3 \mathrm{H}), 2.09-2.17(\mathrm{~m}, 5 \mathrm{H})$, $2.25-2.47(\mathrm{~m}, 3 \mathrm{H}), 2.77(\mathrm{~m}, 1 \mathrm{H}), 3.25-3.36(\mathrm{~m}, 1 \mathrm{H}), 3.67-3.74$ $(\mathrm{m}, 1 \mathrm{H}), 4.11-4.24(\mathrm{~m}, 2 \mathrm{H}), 4.58(\mathrm{~d}, J=7.2 \mathrm{~Hz}), 1 \mathrm{H}], 4.61[(\mathrm{~d}$, $J=7.2 \mathrm{~Hz}, 1 \mathrm{H}), 4.96-5.11(\mathrm{~m}, 2 \mathrm{H}), 5.22(\mathrm{t}, J=9.3 \mathrm{~Hz}, 1 \mathrm{H})$ ppm. ${ }^{13} \mathrm{C}$ NMR $\left(75 \mathrm{MHz}, \mathrm{CDCl}_{3}\right): \delta=20.6,20.7,24.2,24.3,31.8$, $32.0,40.6,40.7,42.3,43.4,48.9,49.0,62.0,62.2,68.2,68.3,69.8$, $69.9,73.7,75.8,77.2,82.7,83.6,169.3,169.4,169.4,170.1,170.1$, 170.6, 170.7, 208.1 ppm. Low-resolution MS: calcd. $m / z 460\left[\mathrm{M}^{+}\right]$, found $461\left[\mathrm{M}^{+}+1\right] . \mathrm{C}_{20} \mathrm{H}_{28} \mathrm{O}_{10} \mathrm{~S}$ (460.5): calcd. C 52.16, H 6.13; found C 51.83, H 5.94 .

3-(2,3,4,6-Tetra- $O$-acetyl- $\beta$-D-glucopyranosylthio)cyclopentanone (24): $[\alpha]_{\mathrm{D}}^{25}=-23(c=1$, chloroform). IR (neat): $\tilde{v}=1748,2853$, $2924 \mathrm{~cm}^{-1} .{ }^{1} \mathrm{H}$ NMR $\left(300 \mathrm{MHz}, \mathrm{CDCl}_{3}\right): \delta=2.01(\mathrm{~s}, 3 \mathrm{H}), 2.03$ $(\mathrm{s}, 3 \mathrm{H}), 2.06(\mathrm{~s}, 3 \mathrm{H}), 2.10-2.13(\mathrm{~m}, 5 \mathrm{H}), 2.19-2.46(\mathrm{~m}, 4 \mathrm{H})$, $2.60-2.72(\mathrm{~m}, 1 \mathrm{H}), 3.67-3.74(\mathrm{~m}, 2 \mathrm{H}), 4.11-4.25(\mathrm{~m}, 2 \mathrm{H}), 4.56$ $[(\mathrm{d}, J=9.9 \mathrm{~Hz}), 4.61(\mathrm{~d}, J=9.9 \mathrm{~Hz}), 1 \mathrm{H}], 5.01-5.14(\mathrm{~m}, 2 \mathrm{H})$, $5.20-5.32(\mathrm{~m}, 1 \mathrm{H}) \mathrm{ppm} .{ }^{13} \mathrm{C} \mathrm{NMR}\left(75 \mathrm{MHz}, \mathrm{CDCl}_{3}\right): \delta=20.5$, 20.6, 20.7, 30.1, 30.7, 37.2, 37.3, 39.5, 40.1, 45.9, 46.7, 61.9, 62.1, $68.1,68.2,69.7,69.9,73.6,75.9,83.2,83.5,169.3,169.4,170.1$, 170.1, 170.5, 215.7, 215.9 ppm. Low-resolution MS: calcd. $\mathrm{m} / \mathrm{z} 446$ $\left[\mathrm{M}^{+}\right]$, found $447\left[\mathrm{M}^{+}+1\right] \cdot \mathrm{C}_{19} \mathrm{H}_{26} \mathrm{O}_{10} \mathrm{~S}$ (446.5): calcd. C 51.11, $\mathrm{H}$ 5.87; found C 51.32, H 5.82 . 
$\boldsymbol{\alpha}, \boldsymbol{\beta}$-Unsaturated $\boldsymbol{\delta}$-Lactone $25:^{[20]} 2,3,6$-Tri- $O$-acetylglucal $(0.300 \mathrm{~g}$, $1.12 \mathrm{mmol}$ ) was placed in a $50-\mathrm{mL}$ round-bottomed flask and cooled to $-20{ }^{\circ} \mathrm{C}$ with the aid of an ice/sodium chloride mixture. $m$ Chloroperbenzoic acid $(0.234 \mathrm{~g}, 1.343 \mathrm{mmol})$ was dissolved in dry chloroform $(4 \mathrm{~mL})$ and added dropwise to the reaction mixture over a period of $10 \mathrm{~min}$, followed by addition of $\mathrm{BF}_{3} \cdot \mathrm{Et}_{2} \mathrm{O}(95 \mu \mathrm{L}$, $0.67 \mathrm{mmol}$ ). Stirring was continued for $1 \mathrm{~h}$, and the reaction mixture was then quenched with ammonia solution $(0.4 \mathrm{~mL})$ and icecold water $(8 \mathrm{~mL})$. After stirring for $10 \mathrm{~min}$, the organic layer was separated and the aqueous layer was washed twice with chloroform $(10 \mathrm{~mL})$. The combined chloroform extracts were washed with water $(10 \mathrm{~mL})$, ammonia solution $(10 \mathrm{~mL})$, again with water $(10 \mathrm{~mL})$, and brine solution $(10 \mathrm{~mL})$. The organic layer was dried with anhydrous sodium sulfate and was filtered. Removal of the solvent gave a viscous oil, which was purified by flash column chromatography on silica gel with ethyl acetate/petroleum ether $(2: 3, \mathrm{v} /$ v) to provide the unsaturated lactone $25(0.155 \mathrm{~g}, 77 \%)$ as a colorless oil. IR (neat): $\tilde{v}=1741,2957 \mathrm{~cm}^{-1} .{ }^{1} \mathrm{H}$ NMR $(300 \mathrm{MHz}$, $\left.\mathrm{CDCl}_{3}\right): \delta=2.10(\mathrm{~s}, 3 \mathrm{H}), 2.15(\mathrm{~s}, 3 \mathrm{H}), 4.31$ (pair of dd, $J=4.2$, $J=12 \mathrm{~Hz}, 2 \mathrm{H}), 4.67(\mathrm{~m}, 1 \mathrm{H}), 5.54(\mathrm{dt}, J=1.5, J=7.4 \mathrm{~Hz}, 1$ $\mathrm{H}), 6.12(\mathrm{~d}, J=9.9 \mathrm{~Hz}, 1 \mathrm{H}), 6.80(\mathrm{dd}, J=1.2, J=9.9 \mathrm{~Hz}, 1 \mathrm{H})$ ppm. ${ }^{13} \mathrm{C} \mathrm{NMR}\left(75 \mathrm{MHz}, \mathrm{CDCl}_{3}\right): 20.4,20.5,61.8,63.2,77.1$, $122.1,143.1,161.0,169.5,170.2 \mathrm{ppm}$.

Synthesis of Compound 27: Tetrathiomolybdate 1 ( $0.134 \mathrm{~g}$, $0.24 \mathrm{mmol}$ ) was added to a solution of dibenzyl disulfide (26, $0.54 \mathrm{~g}, 0.22 \mathrm{mmol})$ in acetonitrile $(3 \mathrm{~mL})$, and the system was stirred at room temperature for $10 \mathrm{~min}$. Unsaturated lactone $\mathbf{2 5}$ $(0.100 \mathrm{~g}, 0.438 \mathrm{mmol})$ in $\mathrm{CH}_{3} \mathrm{CN}(2 \mathrm{~mL})$ was added to this solution, and the reaction mixture was stirred under argon at room temperature for a further $5 \mathrm{~h}$. The solvent was evaporated under reduced pressure, the black residue was extracted with dichloromethane and diethyl ether $(3: 10,13 \mathrm{~mL} \times 4)$ and filtered through a Celite pad, and the filtrate was concentrated. The residue was purified by flash column chromatography on silica gel (eluent: ethyl acetate/petroleum ether, 1:4) to afford compound $27(0.073 \mathrm{~g}, 95 \%)$ as a gummy solid. IR (neat): $\tilde{v}=2919,1746 \mathrm{~cm}^{-1} .{ }^{1} \mathrm{H}$ NMR $\left(300 \mathrm{MHz}, \mathrm{CDCl}_{3}\right): \delta=2.00(\mathrm{~s}, 3 \mathrm{H}), 2.17(\mathrm{~s}, 3 \mathrm{H}), 2.67-2.82(\mathrm{~m}$, $2 \mathrm{H}), 3.20-3.25(\mathrm{~m}, 1 \mathrm{H}), 3.81(\mathrm{~m}, 2 \mathrm{H}), 4.16(\mathrm{dq}, J=4.6, J=$ $12.2 \mathrm{~Hz}, 2 \mathrm{H}), 4.68$ (q, $J=4.3 \mathrm{~Hz}, 1 \mathrm{H}), 5.20(\mathrm{t}, J=3.1 \mathrm{~Hz}, 1 \mathrm{H})$, $7.28-7.36(\mathrm{~m}, 5 \mathrm{H}) \mathrm{ppm} .{ }^{13} \mathrm{C} \mathrm{NMR}\left(75 \mathrm{MHz}, \mathrm{CDCl}_{3}\right): \delta=20.6$, 20.7, 36.1, 36.4, 39.1, 61.7, 70.5, 81.7, 127.7, 128.7, 128.8, 136.8, 169.7, 170.3, 173.6 ppm. Low-resolution MS: calcd. $\mathrm{m} / z 352\left[\mathrm{M}^{+}\right]$, found $375\left[\mathrm{M}^{+}+\right.$Na]. High-resolution MS: calcd. for $\mathrm{C}_{17} \mathrm{H}_{20} \mathrm{O}_{6} \mathrm{~S}$ $+\mathrm{Na} 375.0878$; found 375.0881 .

Bis(methyl 2,3,4-tri- $O$-acetyl-6-deoxy- $\alpha$-D-glucopyranosid-6-yl) Disulfide (28): ${ }^{[20]}$ Tetrathiomolybdate $\mathbf{1}(0.355 \mathrm{~g}, 0.583 \mathrm{mmol})$ was added to a solution of methyl 2,3,4-tri- $O$-acetyl-6-bromo-6-deoxy- $\alpha$ D-glucopyranoside $(0.20 \mathrm{~g}, 0.53 \mathrm{mmol})$ in acetonitrile $(5 \mathrm{~mL})$, and the system was stirred at room temperature for $12 \mathrm{~h}$. The solvent was evaporated under reduced pressure, the black residue was extracted with dichloromethane and diethyl ether $(3: 10,13 \mathrm{~mL} \times 4)$ and filtered through a Celite pad, and the filtrate was concentrated. The residue was purified by flash column chromatography on silica gel (eluent: ethyl acetate/petroleum ether, 2.3) to afford the disulfide $28(0.114 \mathrm{~g}, 85 \%)$ as a colorless solid. M.p. $156-157^{\circ} \mathrm{C}$ (ref. ${ }^{[20]} 157$ $\left.{ }^{\circ} \mathrm{C}\right)$. IR (neat): $\tilde{v}=1735 \mathrm{~cm}^{-1} .{ }^{1} \mathrm{H}$ NMR $\left(300 \mathrm{MHz}, \mathrm{CDCl}_{3}\right): \delta=$ $2.00(\mathrm{~s}, 6 \mathrm{H}), 2.06(\mathrm{~s}, 6 \mathrm{H}), 2.07(\mathrm{~s}, 6 \mathrm{H}), 2.86$ (a pair of dd, $J_{5,6 \mathrm{ax}}=$ $\left.3.2, J_{5,6 \mathrm{eq}}=8.5, J_{6 \mathrm{ax}, \mathrm{eq}}=13.8 \mathrm{~Hz}, 4 \mathrm{H}\right), 3.44(\mathrm{~s}, 6 \mathrm{H}), 4.02(\mathrm{td}$, $\left.J_{5.6 \mathrm{ax}}=3.2, J_{4.5}=8.6 \mathrm{~Hz}, 2 \mathrm{H}\right), 4.81-5.00(\mathrm{~m}, 6 \mathrm{H}), 5.46\left(\mathrm{t}, J_{3,4}=\right.$ $\left.J_{4,5}=9.7 \mathrm{~Hz}, 2 \mathrm{H}\right) \mathrm{ppm} .{ }^{13} \mathrm{C} \mathrm{NMR}\left(75 \mathrm{MHz}, \mathrm{CDCl}_{3}\right): \delta=20.4$, $41.4,55.4,67.5,69.8,70.8,71.7,96.5,169.2,169.6 \mathrm{ppm}$.
Synthesis of Compound 29: Tetrathiomolybdate 1 (0.92 g, $0.15 \mathrm{mmol})$ was added to a solution of disulfide $28(0.10 \mathrm{~g}$, $0.137 \mathrm{mmol})$ in acetonitrile $(3 \mathrm{~mL})$, and the system was stirred at room temperature for $10 \mathrm{~min}$. Unsaturated lactone $25(0.625 \mathrm{~g}$ $0.274 \mathrm{mmol}$ ) was added to this solution, and the reaction mixture was stirred under argon at room temperature for a further $5 \mathrm{~h}$. The solvent was evaporated under reduced pressure, the black residue was extracted with dichloromethane and diethyl ether $(3: 10,13 \mathrm{~mL}$ $\times 4)$ and filtered through a Celite pad, and the filtrate was concentrated. The residue was purified on silica gel (eluent: ethyl acetate/ petroleum ether, 1:1) to afford thiodisaccharide $29(0.069 \mathrm{~g}, 90 \%)$ as a colorless, gummy solid. ${ }^{1} \mathrm{H}$ NMR $\left(300 \mathrm{MHz}, \mathrm{CDCl}_{3}\right): \delta=2.01$ (s, $3 \mathrm{H}), 2.04$ (s, $3 \mathrm{H}), 2.08$ (s, $3 \mathrm{H}), 2.12(\mathrm{~s}, 3 \mathrm{H}), 2.17$ (s, $3 \mathrm{H})$, $2.66-2.80(\mathrm{~m}, 3 \mathrm{H}), 2.94(\mathrm{dd}, J=5.6, J=18 \mathrm{~Hz}, 1 \mathrm{H}), 3.44$ (s, 3 $\mathrm{H}), 3.62(\mathrm{~m}, 1 \mathrm{H}), 3.95(\mathrm{dtd}, J=2.8, J=2.4, J=7.6 \mathrm{~Hz}, 1 \mathrm{H})$, $4.28(\mathrm{dd}, J=4.8, J=6 \mathrm{~Hz}, 2 \mathrm{H}), 4.74(\mathrm{q}, J=4 \mathrm{~Hz}, 1 \mathrm{H}), 4.86$ $(\mathrm{dd}, J=3.6, J=10 \mathrm{~Hz}, 1 \mathrm{H}), 4.92(\mathrm{~d}, J=4 \mathrm{~Hz}, 1 \mathrm{H}), 4.98(\mathrm{t}$, $J=9.8 \mathrm{~Hz}, 1 \mathrm{H}), 5.29(\mathrm{t}, J=3.3 \mathrm{~Hz}, 1 \mathrm{H}), 5.43(\mathrm{t}, J=12.0 \mathrm{~Hz}$, $1 \mathrm{H}) \mathrm{ppm} .{ }^{13} \mathrm{C} \mathrm{NMR}\left(75 \mathrm{MHz}, \mathrm{CDCl}_{3}\right): \delta=20.6,20.7,32.7,33.4$, 39.9, 55.5, 62.7, 66.6, 69.6, 69.7, 70.6, 71.2, 77.6, 96.6, 166.9, 169.7, 169.8, 169.9, 170.0, 170.2 ppm. Low-resolution MS: calcd. $\mathrm{m} / \mathrm{z} 564$ $\left[\mathrm{M}^{+}\right]$, found $587\left[\mathrm{M}^{+}+\mathrm{Na}\right]$. High-resolution $\mathrm{MS}$ : calcd. for $\mathrm{C}_{23} \mathrm{H}_{32} \mathrm{O}_{14} \mathrm{~S}+\mathrm{Na} 587.1410$; found 587.1418.

Synthesis of Compound 31: Tetrathiomolybdate 1 (0.76 g, $0.125 \mathrm{mmol})$ was added to a solution of disulfide $\mathbf{3 0}(0.05 \mathrm{~g}$, $0.113 \mathrm{mmol})$ in acetonitrile $(3 \mathrm{~mL})$, and the system was stirred at room temperature for $10 \mathrm{~min}$. Unsaturated lactone $25(0.515 \mathrm{~g}$, $0.226 \mathrm{mmol}$ ) was added to this solution, and the reaction mixture was stirred under argon at room temperature for a further $5 \mathrm{~h}$. The solvent was evaporated under reduced pressure, the black residue was extracted with dichloromethane and diethyl ether $(3: 10,13 \mathrm{~mL}$ $\times 4)$ and filtered through a Celite pad, and the filtrate was concentrated. The residue was purified on silica gel (eluent: ethyl acetate/ petroleum ether, $3: 7$ ) to afford the (thiogluco)amino acid derivative $31(0.046 \mathrm{~g}, 90 \%)$ as a colorless, gummy solid. ${ }^{1} \mathrm{H}$ NMR $(300 \mathrm{MHz}$, $\left.\mathrm{CDCl}_{3}\right): \delta=1.46(\mathrm{~s}, 9 \mathrm{H}), 2.16(\mathrm{~s}, 3 \mathrm{H}), 2.17(\mathrm{~s}, 3 \mathrm{H}), 2.74(\mathrm{dd}$, $J=10, J=18 \mathrm{~Hz}, 1 \mathrm{H}), 2.88(\mathrm{dd}, J=6, J=14.1 \mathrm{~Hz}, 1 \mathrm{H}), 2.94$ $(\mathrm{dd}, J=5.4, J=17.7 \mathrm{~Hz}, 1 \mathrm{H}), 3.16(\mathrm{dd}, J=4.8, J=10.5 \mathrm{~Hz}, 1$ $\mathrm{H}), 3.55-3.59(\mathrm{~m}, 1 \mathrm{H}), 3.78(\mathrm{~s}, 3 \mathrm{H}), 4.31(\mathrm{qd}, J=4.0, J=$ $12.4 \mathrm{~Hz}, 2 \mathrm{H}), 4.54$ (m, $1 \mathrm{H}), 4.75$ (q, $J=4.4 \mathrm{~Hz}, 1 \mathrm{H}), 5.29-5.33$ $(\mathrm{m}, 2 \mathrm{H}) \mathrm{ppm} .{ }^{13} \mathrm{C} \mathrm{NMR}\left(75 \mathrm{MHz}, \mathrm{CDCl}_{3}\right): \delta=20.6,28.2,33.4$, $34.1,39.0,52.7,52.9,62.9,66.8,77.5,80.5,155.0,166.8,169.8$, 170.2, 170.9 ppm. Low-resolution MS: calcd. $m / z 463\left[\mathrm{M}^{+}\right]$, found $486\left[\mathrm{M}^{+}+\mathrm{Na}\right]$. High-resolution MS: calcd. for $\mathrm{C}_{19} \mathrm{H}_{29} \mathrm{NO}_{10} \mathrm{~S}$ 486.1410; found 486.1416 .

\section{Acknowledgments}

The authors thank the Volkswagen Foundation, Germany, for financial support of this investigation. P. R. S. thanks CSIR, New Delhi for a senior research fellowship.

[1] H. Driquez, ChemBioChem 2001, 2, 311-318.

${ }^{[2]}$ F. Nicotra, Top. Curr. Chem. 1997, 187, 55-83 and references cited therein.

[3] R. Roy, F. Hernandez-Mateo, F. Santoyo-Gonzales, J. Org. Chem. 2000, 65, 8743-8746 and references cited therein.

${ }^{[4]}$ Z. J. Witczak, Curr. Med. Chem. 1999, 6, 165-178.

[5] P. Pasetto, X. Chen, C. M. Brain, R. W. Franck, Chem. Commun. 2001, 81-82.

${ }^{[6]}$ G. Yang, R. W. Frank, R. Bittman, P. Samadder, G. Arthur, Org. Lett. 2001, 3, 197-200 and references cited therein.

[7] R. J. K. Taylor, Chem. Commun. 1999, 217-227 and references cited therein. 
${ }^{[8]}$ T. Zhu, G. J. Boons, Angew. Chem. Int. Ed. 1998, 37, $1898-1900$

[9] [9a] S. Oscarson, in Carbohydrates in Chemistry and Biology (Eds.: B. Ernst, G. W. Hart, P. Sinaÿ), Wiley-VCH, Weinheim, 2000, vol. 1, pp. $93-116$ and references cited therein. ${ }^{[\mathrm{b}] \mathrm{X}}$. Zhu, K. Pachamuthu, R. R. Schmidt, J. Org. Chem. 2003, 68, 5641-5651. [9c] H. Driquez, Top. Curr. Chem. 1997, 187, 85-116. ${ }^{[9 d]}$ U. J. Nilsson, E. J. L. Fournier, O. Hindsgaul, Bioorg. Med. Chem. 1998, 6, 1563-1575. ${ }^{[9 e]}$ M. L Uhrig, O. Varela, Carbohydr. Res. 2002, 337, 2069-2076. ${ }^{[9 f]}$ S. Knapp, D. S. Myers, J. Org. Chem. 2002, 67, 2995-2999.

${ }^{[10]}{ }^{[10 a]}$ D. Horton, Methods Carbohydr. Chem. 1963, 2, 433-437 and references cited therein. ${ }^{[10 b]}$ W. A. Bonner, J. E. Kahn, J. Am. Chem. Soc. 1951, 73, 2241-2245. ${ }^{[10 c]}$ D. Horton, M. L. Wolfrom, J. Org. Chem. 1962, 27, 1794-1800. [10d] S. Chipowsky, Y. C. Lee, Carbohydr. Res. 1973, 31, 229-238. ${ }^{[10 \mathrm{e}]}$ F. M. Ibatullin, S. I. Selivanow, A. G. Shavva, Synthesis 2001, 419-422. ${ }^{[10 f]}$ F. M. Ibatullin, K. A. Shabalin, J. V. Jänis, A. G. Shavva, Tetrahedron Lett. 2003, 44, 7961-7964.

[11] [11a] Review: B. J. Conchie, G. A. Levvy, C. A. Mrach, $A d v$. Carbohydr. Chem. Biochem. 1957, 12, 157-187. [11b] K. L. Matta, R. N. Girotra, J. J. Barlow, Carbohydr. Res. 1975, 43, 101-109. ${ }^{[11 \mathrm{c}]}$ B. J. Conchie, G. A. Levvy, Methods Carbohydr. Chem. 1963, 2, 335-337. [11d] M. Apparu, M. Blanc-Muesser, J. Defaye, H. Driquez, Can. J. Chem. 1981, 59, 314-323. [11e] E. Yu. Tsvetkov, N. E. Byramova, L. V. Backinovsky, Carbohydr. Res. 1983, 115, 254-258.

[12] [12a] P. L. Durette, T. Y. Shen, Carbohydr. Res. 1980, 81, 261-274. ${ }^{[12 b]}$ R. T. Lee, Y. C. Lee, Carbohydr. Res. 1982, 101, 49-55. ${ }^{[12 c]}$ A. Hasegawa, M. Morita, Y. Kojima, H. Ishida, M. Kiso, Carbohydr. Res. 1991, 214, 43-53. [12d] J. Defaye, J. M. Gluillot, Carbohydr. Res. 1994, 253, 185-194. ${ }^{[12 \mathrm{e}]}$ M. O. Contour-Galcera, J. M. Guillot, C. Ortiz-Mellet, F. Pflieger-
Carara, J. Defaye, J. Gelas, Carbohydr. Res. 1996, 281, 99-118. ${ }^{[12 f]}$ M. O. Contour-Galcera, Y. Ding, C. Ortiz-Mellet, J. Defaye, Carbohydr. Res. 1996, 281, 119-128. [12g] G. Schwarzmann, P. Hofmann, U. Putz, Carbohydr. Res. 1997, 304, 43-52.

${ }^{[13]}$ Review: D. Horton, D. H. Hutson, Adv. Carbohydr. Chem. Biochem. 1963, 18, 123-199.

[14] [14a] R. R. Schmidt, M. Stumpp, Liebigs Ann. Chem. 1983, 1249-1256. ${ }^{[14 b]}$ J. S. Andrews, B. M. Pinto, Carbohydr. Res. 1995, 270, 51-62. ${ }^{[14 \mathrm{c}]}$ T. Eisele, A. Toepfer, G. Kretzschmar, R. R. Schmidt, Tetrahedron Lett. 1996, 37, 1389-1392.

${ }^{[15]}$ Z.-J. Li, P.-Liu, Z.-J. Li, D.-X. Fqiu, M.-S. Cai, Synth. Commun. 1990, 20, 2169-2175.

${ }^{[16]}$ P. Li, L. Sun, D. W. Landry, K. Zhao, Carbohydr. Res. 1995, $275,179-184$.

${ }^{[17]}$ I. Fokt, W. Szeja, Carbohydr. Res. 1992, 232, 169-175.

${ }^{[18]}$ J. M. Lacombe, N. Rakotomanomana, A. A. Pavia, Tetrahedron Lett. 1988, 29, 4293-4296.

${ }^{[19]}$ K. R. Prabhu, N. Devan, S. Chandrasekaran, Synlett 2002, $1762-1778$

${ }^{[20]}$ D. Bhar, S. Chandrasekaran, Carbohydr. Res. 1997, 301, 221-224.

${ }^{[21]}$ K. R. Prabhu, P. Sivanand, S. Chandrasekaran, Angew. Chem. Int. Ed. 2000, 39, 4316-4319.

${ }^{[22]}$ R. L. Whistler, A. K. M. Anisuzzaman, Methods in Carbohydrate Chemistry, Academic Press, Inc., New York, 1980, vol. VIII, p. 227-231.

${ }^{[23]}$ D. Miljkovic, N. Vukojevic, D. Medakovic, Carbohydr. Res. 1989, 193, 275-278.

${ }^{[24]}$ F. W. Lichtenthaler, K. Lorenz, W. M. Yong, Tetrahedron Lett. 1987, 28, 47-50.

${ }^{[25]}$ D. H. R Barton, J. Camara, X. Cheng, S. D. Gero, J. Cs. Jaszberenyi, B. Quiclet-Sire, Tetrahedron 1992, 48, 9261-9276. 